\title{
Acceptability and Technical Feasibility of an Electrical Wiring Installation Trainer
}

\author{
Dr. Jeff Galapon Pereyras ${ }^{1}$ \\ ${ }^{1}$ Pangasinan State University - Lingayen Campus, Philippines, jpereyras@psu.edu.ph
}

\begin{abstract}
The use of mock-up trainer as an instructional material is very significant in the cycle of teaching-learning in developing technical skills and exercises, certainly in building and residential wiring installation and maintenance to guarantee the learners quality education in an industrial-like scenario. This study employed a descriptive method of research by determining the trainers' acceptability using the variables functionality, usefulness, safety, and maintenance, and it is technical feasibly using the variables - validity, reliability, and replicability by the electrical experts from the academe and the industry. After series of demonstrations and technology assessments of the respondents, the said trainer was rated by both experts from the academe particularly teaching electrical technology and engineering, and the electrical industry acceptable and technical feasible on all the criteria in the given instrument. With this trainer present in the shop-laboratory, learners can develop their expertise by accomplishing such tasks because it is along-side the real market of building and industrial wiring installation and maintenance.
\end{abstract}

Key words: Acceptability, Electrical wiring installation, Technical feasibility, Trainer.

\section{INTRODUCTION}

Throughout the background of electrical power networks, there is a vital operation that allows those working in the electrical power field to fulfill the specifications of a particular device [1]. The electrical wiring installation trainer offers learners with the knowledge and skills of the elective subject, as well as instructor presentations and assessment of student results during the laboratory period. The trainer board was 2.4 meters by 2.4 meters by 0.02 -meter in size. This was economical, lightweight, and compact. It includes a circuit breaker, a number of service boxes, a number of junction boxes, a number of single switches, a number of three-way switches, a number of convenient sockets, a number of baseboards, a number of receptacles, a number of Light
Emitting Diode (LED) lights, a number of Flexi hoses, and a number of u-clamps. All system connections have been opened and exposed to quick deployment and adaptation. It was fitted with a low-current circuit breaker to promote stable operation and prevent harm to all equipment. It was proficient in treating, troubleshooting, and commissioning domestic, business, and industrial-like electrical wiring installations. The electrical wiring installation trainer was operated by a 220 V-AC (Volts-Alternative Current) power supply and assisted by an instructional module in the electrical wiring system. The Industrial Design (ID) patent and the Utility Model (UM) patent was issued to the electrical wiring installation trainer by the Intellectual Property Office of the Philippines [2] which was designed and developed by the author of this paper.

The Technology Acceptance Model (TAM) is a philosophy that describes how people embrace and utilize technology. TAM deals more directly with the calculation of the acceptability of the information system. The primary aim of this model is to assess the acceptability of the system and to determine the changes that need to be made to the process to render it available to end-users. This concept recommends that the acceptability of the information system is defined by two main factors: perceived usefulness and perceived ease of use. Perceived usefulness is identified as the point to which an individual expects that the usage of a tool will increase its performance. Perceived ease of use concerns the ranking at which the user thinks that the usage of the system should be uncomplicated. Numerous empirical analysis found that perceived usefulness and perceived ease of use can be interpreted as two separate dimensions [3].

Technical feasibility is the phase of evaluating the technological design and architecture of a certain project or a product. The technical feasibility test is particularly useful in screening and prioritizing technologies. This screening process aims to fast track the shortlisting of technologies for assessment, thus saving time, money, and effort in evaluating the technologies which are not technically feasible in the first place. The evaluation shall determine the validity, reliability, and replicability of the technologies.

The Pangasinan State University (PSU) -Lingayen Campus, in particular the College of Technology (CT), is providing a 
Jeff Galapon Pereyras, International Journal of Advanced Trends in Computer Science and Engineering, 9(3), May - June 2020, 2995- 2999

degree Bachelor in Industrial Technology (BIT) in nine (9) main areas of specialty of which Electrical Technology is one of its flagship program [4]. Since then, the form of directions provided in this course, building wiring installation, has been taught solely by design, taking into account the fact that the subject has a portion of the laboratory structure. As a consequence, students who have taken up this topic can visualize designing wiring systems through internet videos and later via their labs and field trips to several electrical and manufacturing firms. To enhance the accuracy of the student learning results of the BIT Major in Electrical Technology, the trainer's maker aims to assess its acceptability and its technical feasibility for the electrical engineers, technologists, and electricians from the academe and the industry sector.

\section{LITERATURE REVIEW}

In the research of Pereyras, 2019, entitled "Computerized touchscreen student voting system for universities and colleges", computerized student touchscreen voting method defined as a computer-mediated voting system in which the student-voters render their choices using a touchscreen machine. This study was done to test the efficacy of the current student voting system at the colleges and universities, especially at the Pangasinan State University - Lingayen Campus, where it has been sampled as a basis for implementing a computerized touchscreen student voting system. Furthermore, the analysis also assessed the acceptability of students of the new computerized interactive electronic voting method. Upon close consideration of the results and conclusions of the report, it is strongly suggested that the administration of the numerous colleges and universities, in particular the trial university, the Pangasinan State University - Lingayen Campus, with the consent of the Office of the Student Affairs and the Student Council of the University, to follow the proposed computerized touchscreen program. [5].

On the study of Tonido, 2012, entitled "Development and Acceptability of Low-Cost Electro-Tech Trainer Board, Series-E09", it notes that the utilization of training device and appropriate instructional materials are essential in the teaching and learning cycle in training and education, especially in the Control of Industrial Motors, to guarantee the standard of learners training in an industrial-like setting. The said study utilized both developmental and descriptive methods of research. The Low-Cost Electro-Tech Trainer Board, Series -E09 was planned, assembled, and assessed its acceptability on the following criteria: functionality, usefulness, safety, and maintenance by electrical technologist and selected electrical students as respondents. The trainer kit was built from indigenous produced products that make it economical than the trainer board already sold commercially. It achieved its utility based on its architecture. After multiple checks and modifications, it was considered to be highly appropriate as assessed by the chosen respondents. This research suggests that the trainer kit be used for teaching and learning practices and developed to be sold in the market [6].

Another study of Pereyras, 2019, entitled "Feasibility Study on the Installation of Solar Photovoltaic Rooftop System for the Pangasinan State University", verify that Pangasinan State University (PSU) in particular Urdaneta City Campus, Sta. Maria Campus, Binmaley Campus, and Infanta Campus consume more than 590,000 kilowatt-hours $(\mathrm{kWh})$ of power each year. The PSU is hunting for opportunities to reduce the costs and environmental effects of its operations. One viable solution is the usage of solar resources for the production of electricity at the four (4) campuses. Solar electricity, though, is, in fact, a difficult problem for an enormous undertaking. Considering the time and options before beginning a project of this size, it is important to save resources and energy in the years yet to come. This study is projected to check the possibility and related remunerations of solar photovoltaic (PV) roofing system setting up relative to the generating potential on its campuses. Solar photovoltaic datasheets were calculated to quantify the cumulative expected sum of electrical power every solar device will produce annually. Cost details were used to determine the expense of each device from the Solar Philippines and the expense of power per kilowatt-hour from every renewable energy network and the various delivery systems for every campus. Additionally, Campuses space and land, and energy consumption records, specifically from the University Engineer Office, have been utilized to test the scale and capability of the necessary solar energy installations and the usability of campus land and space. Also, meetings and presentations to the Campus Executive Officers, Physical Facilities Administrator, and Campus Accountant were done to discuss the non-monetary and of course the financial effects of solar power utilization at the university. A solar photovoltaic rooftop device is the cheapest option, with a payback time of nine and a half years (9.5) and a price of 10 Philippine Pesos (PhP) per kilowatt-hour. For 3,360 square meters of roof area eligible for usage, up to $336,000 \mathrm{kWh}$ or $57 \%$ of the four (4) campuses, solar power will be utilized in 2018. Non-monetary incentives include minimized environmental costs, training and career resources, and a sign of efficiency, carbon protection, and brand advertisement. The University can't generate $100 \%$ of its electricity from on-campus solar power installation. In comparison, solar power is somewhat costly relative to traditional energy prices. Getting a power purchase arrangement with Solar Philippines Inc. (SPI) and the PSU, however, will build a solar PV roofing device at no expense at all and would also have a net profit of 38 percent on a monthly energy bill, despite the non-monetary remunerations, The construction of a solar PV roofing device on the aforementioned four (4) campuses should be considered by the PSU Administration [7].

Technology Assessment Protocol (TAP) was developed by the Philippines Council for Agriculture, Forestry, and Natural Resources Research and Development (PCARRD) under the 
Department of Science and Technology (DOST) to see to it the technologies it has generated are commercialized and utilized by the intended end-users. The TAP is a thorough yet user-friendly process fro evaluating the merits of technology before its commercialization. It creates a solid foundation for identifying limitations and threats and thus sets the way for product development, introduction, and commercialization or repackaging of innovations that are already being commercialized. One of the objectives of TAP was to determine the degree of maturity of technologies using technical feasibility. Technical Feasibility Test answers the following areas of concern, validity - has a specific basis for the technology been established? are there reports, write-ups, and conference papers written and published? reliability - can the laboratory results / on-station trial results be reproduced in field conditions? and, replicability - can other interested groups, technical and commercial, produce approximately the same results? [8].

All of those above-reviewed articles refer to current research as it aimed to recognize the value of training facilities and the need for an innovative instructional mock-up device.

\section{OBJECTIVES OF THE STUDY}

The key purpose of this study was to distinguish the acceptability electrical wiring installation trainer along with the areas of functionality, usefulness, safety and maintenance, and the trainers' technical feasibility along with the areas of validity, reliability, and replicability by the electrical experts from the academe and the industry for use in learning building and industrial wiring installation and maintenance in BIT - Electrical Technology courses as an instructional mockup device.

\section{METHODOLOGY}

This study employed a descriptive research method. To conclude the acceptability and the technical feasibility of the electrical wiring installation trainer, the said mock-up device was exhibited and demonstrated for technology assessment [9] to the five (5) faculty members of the College of Technology of PSU - Lingayen Campus particularly in Electrical Technology and Electronics Technology department, and to the two (2) Electrical Technology Professors at the Technical Education and Skills Development Authority (TESDA) Pangasinan Provincial Office (PO) Training Center, and to the three (3) Electrical Technology Professor of Pangasinan School of Arts and Trade (PSAT) - TESDA which serves as the experts in the academe-respondents, and to the five (5) Electrical Engineers, Electrical Technologist and Electricians of Central Pangasinan Electric Cooperative (CENPELCO) at San Carlos City, and also to the five (5) Electrical Engineers, Electrical Technologist and Electricians of Dagupan Electric Corporation (DECORP) which serves as the experts in the industry-respondents. On the acceptability test of the electrical wiring installation trainer, an instrument was adopted from the study of Tonido (2012) and was given to the said respondents, and on the technical feasibility test, an instrument was also adopted from the Technology Assessment Protocol developed by the DOST-PCARRD was also given to the same set of respondents.

\section{RESULTS AND DISCUSSION}

The average weighted mean for the acceptability of an electrical wiring installation trainer in terms of functionality, usefulness, safety, and maintenance, as viewed by the experts from the academe-respondents was presented in Table 1 . Centered on the experiences of certain respondents from the academe, the Functionality criterion had a weighted average of 4.4 and a verbal definition of "Very Functional", for Usefulness, with an overall weighted average of 4.3 and a verbal meaning of "Very Useful", and for Safety, with an overall weighted average of 4.4 and with a verbal definition of "Very Safe". Lastly, for Maintenance, it had an overall weighted average of 4.4 and a verbal meaning of "Very Easy to Maintain".

Table 1: Acceptability of the Electrical Wiring Installation Trainer as perceived by the Experts from the Academe

\begin{tabular}{|l|c|c|c|c|c|c|c|c|c|c|c|c|}
\hline \multicolumn{1}{|c|}{ Variable } & \multicolumn{4}{|c|}{ PSU-CT } & \multicolumn{4}{c|}{ PSAT-TESDA } & TESDA-PO & Wx & VI \\
\hline Functionality & 5 & 4 & 5 & 4 & 5 & 4 & 4 & 5 & 4 & 4 & 4.4 & VF \\
\hline Usefulness & 5 & 4 & 5 & 5 & 4 & 4 & 4 & 4 & 4 & 4 & 4.3 & VU \\
\hline Safety & 4 & 5 & 5 & 5 & 5 & 4 & 4 & 4 & 4 & 4 & 4.4 & VS \\
\hline Maintenace & 5 & 4 & 5 & 4 & 5 & 4 & 5 & 4 & 4 & 4 & 4.4 & VEM \\
\hline
\end{tabular}

The overall weighted mean for the acceptability of an electrical wiring installation trainer in terms of functionality, usefulness, safety, and maintenance, as assessed by the experts from the industry-respondents was presented in Table 2. Centered on the opinions of said experts in the industry-respondents, criterion Functionality has a weighted average of 4.3 and with a verbal interpretation of "Very Functional", for Usefulness, with an overall weighted average of 4.2 and with a verbal meaning of "Very Useful", and for Safety, with an overall weighted average of 4.4 and with a verbal interpretation of "Very Safe". Lastly, for Maintenance, it received a weighted average of 4.3 and with a verbal definition of "Very Easy to Maintain".

Table 2: Acceptability of the Electrical Wiring Installation Trainer as perceived by the Experts from the Industry

\begin{tabular}{|l|c|c|c|c|c|c|c|c|c|c|c|c|}
\hline \multicolumn{1}{|c|}{ Variable } & \multicolumn{5}{|c|}{ CENPELCO } & \multicolumn{5}{c|}{ DECORP } & Wx & VI \\
\hline Functionality & 5 & 4 & 4 & 4 & 4 & 5 & 4 & 4 & 4 & 5 & 4.3 & VF \\
\hline Usefulness & 5 & 4 & 4 & 4 & 4 & 4 & 4 & 4 & 4 & 5 & 4.2 & VU \\
\hline Safety & 5 & 4 & 4 & 4 & 5 & 5 & 4 & 4 & 4 & 5 & 4.4 & VS \\
\hline Maintenace & 5 & 4 & 4 & 4 & 4 & 4 & 4 & 4 & 5 & 5 & 4.3 & VEM \\
\hline
\end{tabular}

As shown in Table 1 and Table 2, the functionality of the mock-up trainer as perceived by both experts from the academe-respondents and experts from the industry-respondents received an overall weighted average 
score of 4.4 and 4.3 correspondingly and transcribed as "Very Functional". This implies that the teacher may execute all the relevant tasks specified in the instruction module being developed by the author and the trainer functions appropriately. The trainer also adheres to meet the related set of electrical functionality standards. Moreover, the trainer also produces accurate results and functions without encountering any problems.

As shown in Table 1 and Table 2, on the Usefulness of the mock-up trainer as perceived by both experts from the academe-respondents and experts from the industry-respondents received an overall weighted average score of 4.3 and 4.2 correspondingly and transcribed as "Very Useful". This implies that the trainer particularly its material components can easily be installed on its trainer board. And with its simple and user- friendly electrical layout, and different activities that can be performed in the trainer can easily be learned.

With regards to the Safety criterion, both groups of respondents rated the said trainer device "Very Safe". This is evident in Table 1 and Table 2. This manifests that the trainer can also be utilized by the learners even if the learners do not have technical expertise because it is equipped with a low current circuit breaker which serves as a protective device of the trainer.

Lastly, on the criterion Maintenance, the table above also shows that maintainability received an overall weighted average of 4.4 and 4.3 as viewed by the two groups of respondents which mean that the trainer is also "Very Easy to Maintain". This means that trainers can be easily maintained and can be modified in terms of its electrical layout. Electrical faults and errors can also be detected and can be verified effortlessly.

The overall weighted mean for the technical feasibility of an electrical wiring installation trainer in terms of validity, reliability, and replicability as perceived by the experts from the academe-respondents was presented in Table 3. Based on the assessment of the said respondents from the academe, all the criteria Validity, Reliability and Replicability got a weighted average of 4.4 which has a verbal meaning of "Very Valid", "Very Reliable" and "Very Replicable" respectively.

Table 3: Technical Feasibility of the Electrical Wiring Installation Trainer as perceived by the

Experts from the Academe

\begin{tabular}{|l|c|c|c|c|c|c|c|c|c|c|c|c|}
\hline \multicolumn{1}{|c|}{ Variable } & \multicolumn{4}{|c|}{ PSU-CT } & \multicolumn{3}{|c|}{ PSAT-IESDA } & TESDA-PO & Wx & VI \\
\hline Validity & 5 & 4 & 5 & 4 & 5 & 5 & 4 & 4 & 4 & 4 & 4.4 & VL \\
\hline Reliability & 5 & 5 & 4 & 4 & 5 & 4 & 5 & 4 & 4 & 4 & 4.4 & VR \\
\hline Replicabity & 4 & 5 & 5 & 5 & 4 & 4 & 5 & 4 & 4 & 4 & 4.4 & VR \\
\hline
\end{tabular}

The weighted average for the technical feasibility of an electrical wiring installation trainer in terms of validity, reliability, and replicability, as perceived by the experts from the industry-respondents was presented in Table 4. Based on the impressions of the said respondents from the industry, the criterion Validity it got a weighted average of 4.3 and with a verbal definition of "Very Valid", for Reliability, with an overall weighted average of 4.2 and with a verbal meaning of "Very Reliable", and lastly for Replicability, with an overall weighted average of 4.4 and with a verbal definition of "Very Replicable”.

Table 4: Technical Feasibility of the Electrical Wiring Installation Trainer as perceived by the Experts from the Industry

\begin{tabular}{|l|c|c|c|c|c|c|c|c|c|c|c|c|}
\hline \multicolumn{1}{|c|}{ Variable } & \multicolumn{5}{|c|}{ CENPELCO } & \multicolumn{5}{c|}{ DECORP } & VI \\
\hline Validity & 4 & 5 & 4 & 4 & 4 & 5 & 4 & 4 & 4 & 5 & 4.3 & VL \\
\hline Reliability & 5 & 4 & 4 & 4 & 4 & 4 & 4 & 4 & 4 & 5 & 4.2 & VR \\
\hline Replicabity & 4 & 4 & 5 & 4 & 4 & 5 & 5 & 4 & 4 & 5 & 4.4 & VR \\
\hline
\end{tabular}

As shown in Table 3 and Table 4, on the Validity of the mock-up trainer as perceived by both experts from the academe-respondents and experts from the industry-respondents received an overall weighted mean score of 4.4 and 4.3 correspondingly and transcribed as "Very Valid". This implies that the trainer has the specific basis for the technology that has been established, and there parallel researched and studies that have been presented in the research for and conference, and accepted for publications.

On the criterion Reliability, the two groups of expert-respondents rated the reliability of the trainer "Very Reliable". This simply means that the trainer device can function the same in the shop-laboratory and on actual residential building wiring installations. Activities listed in the instructional module that can be performed using the electrical wiring installation trainer are parallel or the same in that of the actual building and industrial wiring installation and maintenance.

Lastly on criterion Replicability, the table above also shows that replicability received an overall weighted average of 4.4 and 4.3 as viewed by the two groups of respondents which mean that the trainer is also "Very Replicable". This means that trainers can be reproduced by other interested groups, technical and commercial, and has approximately the same functions. But as of the moment, the said trainer was already patented by the Intellectual Property Office of the Philippines to the author of this paper and owns the all the rights on the design and patent of the electrical wiring installation trainer.

The features of the newly developed trainer may serve as a standard template that can be used for different applications in residential, building, and even industrial wiring installations and maintenance [10].

\section{CONCLUSION}

Based on the responses of the expert from the academe and the electrical industry, it is concluded that the electrical 
wiring installation trainer was very acceptable and very technically feasible. This means the results of the evaluation corresponds that the study met its objectives. The expert-respondents agreed that the trainer device is very functional, very useful, very safe, very easy to use, very valid, very reliable, and very replicable. This implies that the mock-up trainer can help to provide the students' learning experience and understanding of the subject and course as well as instructor presentations and assessment of student results during the laboratory period.

\section{RECOMMENDATIONS}

Taking into consideration, the report points out that it draws on the results of this analysis, this research proposed the following that the developed trainer be used for teaching-learning tasks such as in higher education institutions and technical and vocational skills development centers who use to offer electrical technology, and the patented trainer be produced for commercialization purposes.

\section{ACKNOWLEDGMENT}

Thank you so much to the Pangasinan State University Lingayen Campus. Thank you to Dr. Catalina Platon, University Head of Intellectual Property and Research, for assistance in registering the utility model at the Intellectual Property Office of the Philippines. Finally, a huge thank you and thanks to Dr. Randy Joy M. Ventayen for his mentoring and supporting the author.

\section{REFERENCES}

[1] Torrizo, L. F., \& Africa, A. D. (2019). Next-hour electrical load forecasting using an artificial neural network: Applicability in the Philippines. International Journal of Advanced Trends in Computer Science and Engineering, 8(3), 831-835. https://doi.org/10.30534/ijatcse/2019/77832019.

[2] Pereyras, J. G. (2020). Development of an electrical wiring installation trainer. International Journal of Scientific and Technology Research, 9(3).4458-4461.

[3] Technology acceptance model. (n.d.). https://edutechwiki.unige.ch/en/Technology_acceptanc e_model.

[4] Pereyras, J. G. (2019). Development of Electro-pneumatic Control Trainer. Asian Journal of Multidisciplinary Studies, 2(2).

[5] Pereyras, J. G. (2019). A Computerized Touchscreen Student Voting System for the Universities and Colleges. Asian Journal of Business and Technology Studies, 2(1).

[6] Tonido, A. R. (2012). Development and Acceptability of Low-Cost Electro-Tech Trainer Board, Series-E09. The University of Rizal System.

[7] Pereyras, J. G. (2019). Feasibility Study on the Installation of Solar Photovoltaic Rooftop System for the Pangasinan State University. Asian Journal of Multidisciplinary Studies, 2(1).

[8] Philippine Council for Agriculture, Forestry and Natural Resources Research and Development. Technology Assessment Protocol. Los Baños, Laguna. PCARRD, 2001. 36p. - (Information Bulletin No. 219).

[9] Bulan, L. A., Maderazo, L. R., \& Asi, P. B. (2019). Design and development of microcontroller-based air conditioning units controller as input to energy conservation for the University of Batangas. International Journal of Advanced Trends in Computer Science and Engineering, 8(4), 1020-1025. https://doi.org/10.30534/ijatcse/2019/06842019.

[10] Gustilo, R. C., Danting, J. R. A., De Vera, L. P. M., Ramos, B. J., \& Villanueva, L. (2019). Design of multi-purpose heavy-duty scanning robot with spray mechanism. International Journal of Advanced Trends in Computer Science and Engineering, 8(3), 561-566. https://doi.org/10.30534/ijatcse/2019/35832019. 